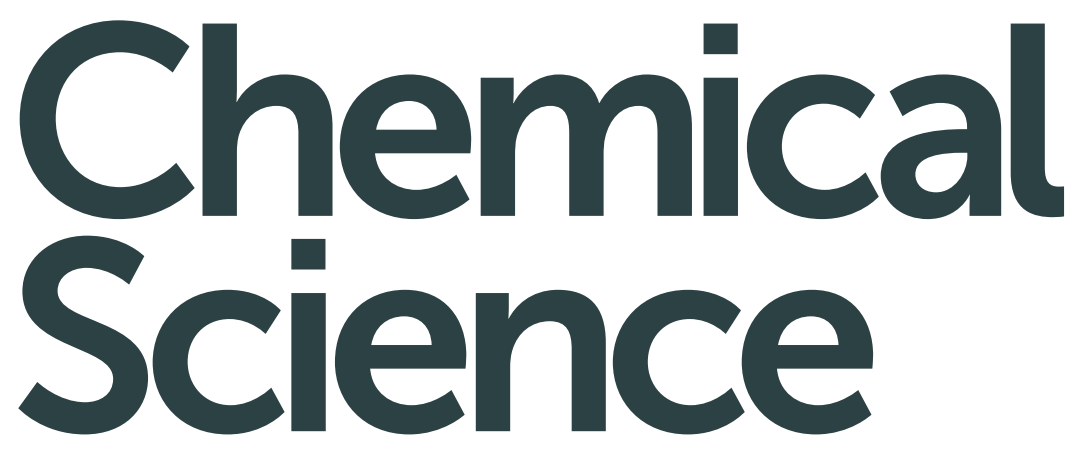

rsc.li/chemical-science

6
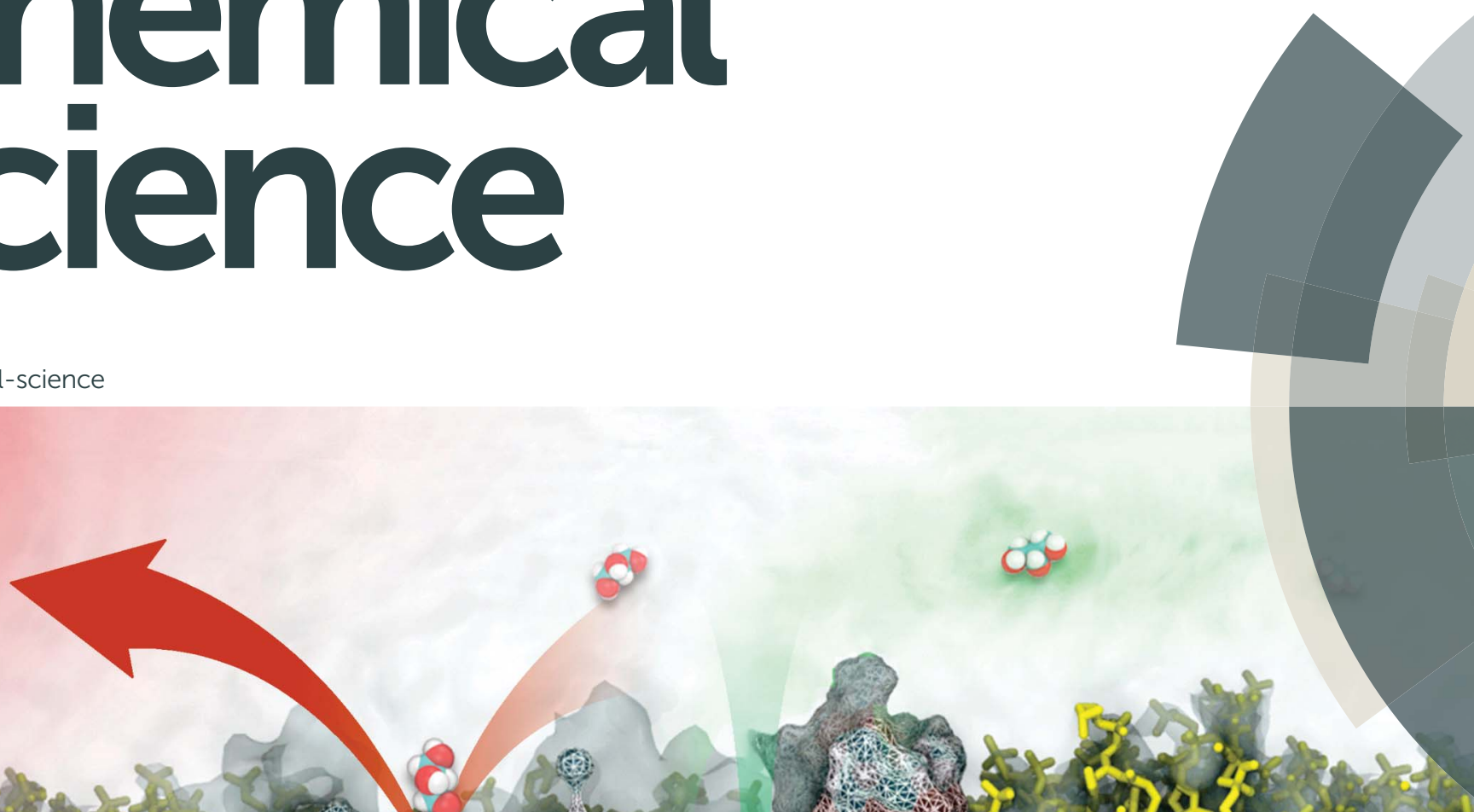
3. 1) (5) Mite (4)

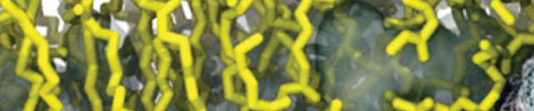
3.12. I. Th

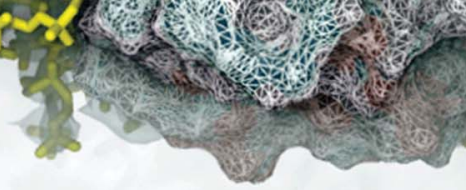
(1)
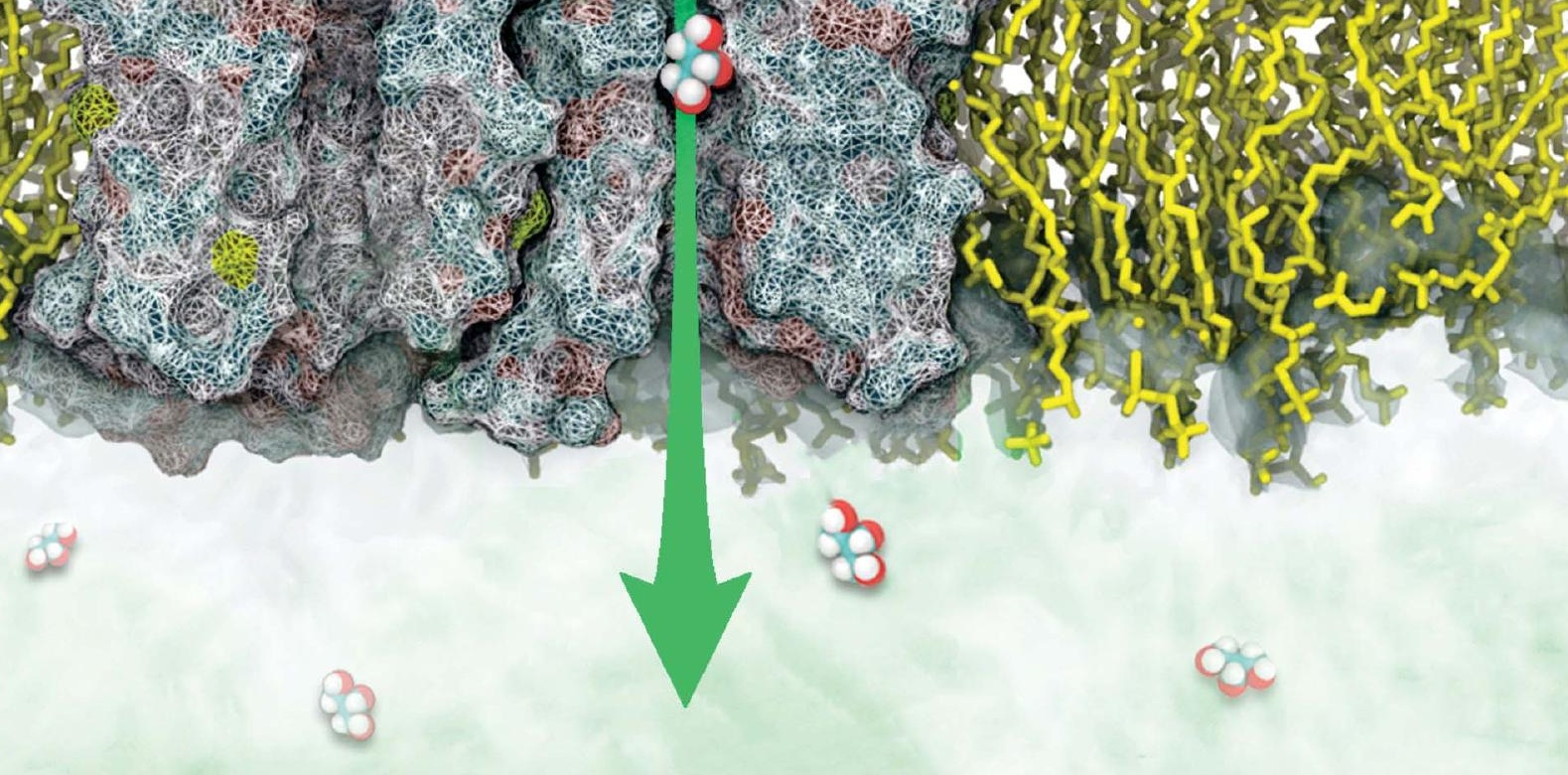

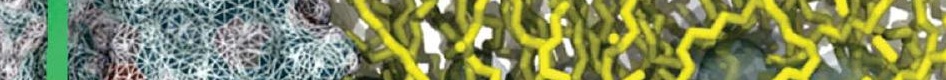

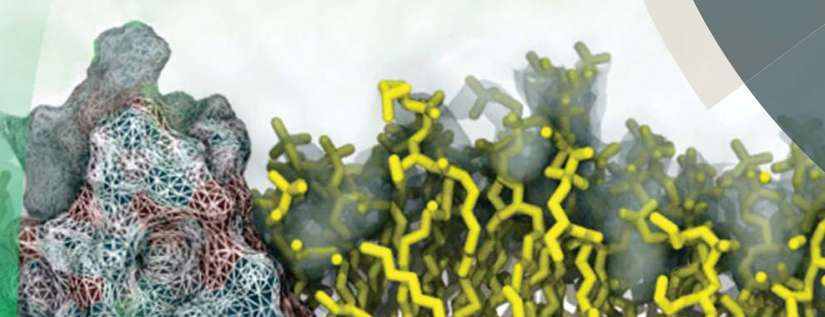

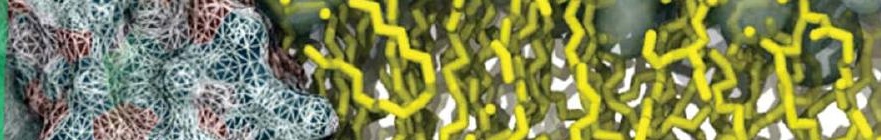

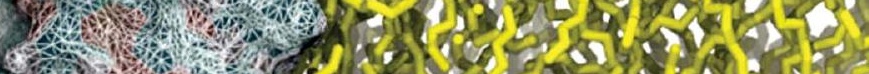

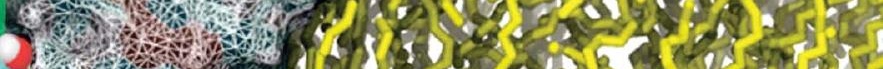

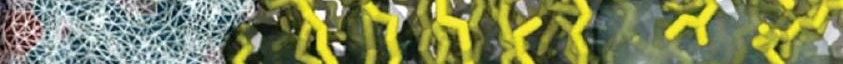

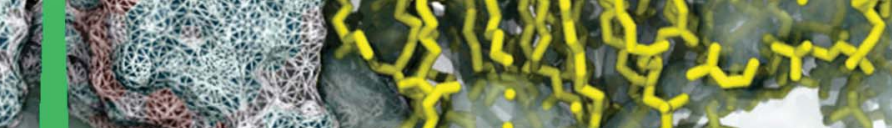
2. (Fos

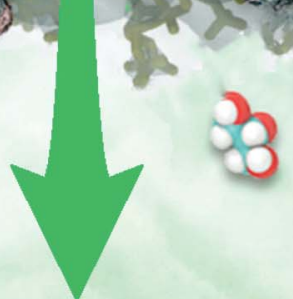

ISSN 2041-6539

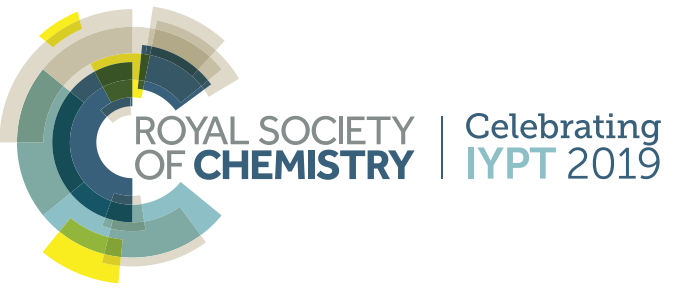

EDGE ARTICLE

Jingwei Weng, Wenning Wang et al.

Glycerol transport through the aquaglyceroporin GlpF bridging dynamics and kinetics with atomic simulation 
Check for updates

Cite this: Chem. Sci., 2019, 10, 6957

๑ All publication charges for this article have been paid for by the Royal Society of Chemistry

Received 6th April 2019

Accepted 17th June 2019

DOI: $10.1039 / c 9 s c 01690 b$

rsc.li/chemical-science

\section{Glycerol transport through the aquaglyceroporin GlpF: bridging dynamics and kinetics with atomic simulation $\uparrow$}

\author{
Dongdong Wang, Jingwei Weng (iD * and Wenning Wang (iD *
}

\begin{abstract}
The aquaglyceroporin GlpF is a member of the aquaporin family. It selectively conducts small molecules, such as glycerol, across the cell membrane under a concentration gradient of the substrate. Atomistic molecular dynamics (MD) simulation would provide great insight into the substrate transport mechanism of GlpF and membrane channels alike. Ideally, non-equilibrium simulations under various concentration gradients of glycerol are desired to emulate the transportation in cells, but this kind of simulation is difficult due to a complicated system setup and high computational cost. Here, we present a new strategy to extract non-equilibrium kinetic information from equilibrium MD simulation. We first performed long-time (totally $22.5 \mu \mathrm{s}$ ) multi-copy equilibrium MD simulations of glycerol conduction through GlpF. Tens of times the spontaneous permeation of glycerol through GlpF was observed, allowing us to elucidate the detailed mechanism of the stereoselectivity for glycerol. Then we employed Markov state model (MSM) analysis of the MD trajectories to identify the intermediate states during glycerol transport and calculate the inter-state transition rate constants. Based on the results of MSM analysis, we built the kinetic models of glycerol transport and calculated the glycerol fluxes under various concentration gradients by solving the master equations. The results agree well with the experimental measurement at a certain glycerol concentration, and provide holistic information on the glycerol conduction capacity of GlpF. Our work demonstrates that long-time atomistic MD simulations can now bridge the microscopic dynamics and the kinetic description of substance transport through membrane channels, hopefully facilitating the engineering of new selective channels for various molecules.
\end{abstract}

\section{Introduction}

Facilitated translocation of molecules through channels and pores is important for transmembrane transport in biological systems. Molecular dynamics (MD) simulation is a powerful tool for the detailed mechanistic study of channel transport., ${ }^{1,2}$ However, realistic simulation of non-equilibrium substrate translocation through channels under a concentration gradient across the membrane is not straightforward. Due to the periodic conditions of the simulation system, explicitly maintaining a substrate concentration difference requires a large simulation

Department of Chemistry, Institutes of Biomedical Sciences, Multiscale Research Institute of Complex Systems, Fudan University, Shanghai, P. R. China. E-mail: jwweng@fudan.edu.cn; wnwang@fudan.edu.cn

$\dagger$ Electronic supplementary information (ESI) available: Convergence of the MD simulation, state-dependent PMFs of different conformational and prochiral states of glycerol, inter-state transition probability after a time span of $10 \mathrm{ps}$ versus the position of glycerol along the pore, projections of 39 successful passages through the major barrier region, MSM validation, statistics of simultaneously occupied sites inside the channel at various glycerol concentrations, summary of the dwell time of spontaneous glycerol conduction events, and the rate constants of the transitions between the eight macrostates obtained from the 202-state Markov model. See DOI: 10.1039/c9sc01690b system and/or special setups., ${ }^{3,4}$ On the other hand, simulating sustained steady states under various concentration differences is computationally expensive. Alternatively, substrate translocation could be simulated under equilibrium conditions, and kinetic information, such as transport flux and rate constant, is estimated based on the potential of mean force (PMF) of substrate permeation and transition state theory. Although the pore axis is a natural reaction coordinate for substrate transport, generally PMF does not govern dynamics, especially in the case of wide pores. Furthermore, as the interaction between the substrate and the channel entails multiple energy barriers and substrate binding sites inside the channel, the transport process could not be approximated by an elementary reaction model. Alternatively, the transport kinetics is better described by a discrete-state Markovian model. However, using PMF profiles to discretize the state space along the translocation pathway is highly arbitrary. Recently, a more rigorous method, the Markov state model (MSM) method, ${ }^{5-12}$ has been widely applied to MD simulations of biomolecules. Combined with large scale multicopy MD simulations, the MSM method has been successfully applied to kinetic analysis of problems like protein folding, and various conformational changes of proteins. ${ }^{5,13-18}$ By using MSM analysis, we could obtain more reasonable discretization of the 
state space and rate constants, based on which the nonequilibrium steady-state kinetics could be calculated through constructing a discrete-state kinetic model and solving master equations under various boundary conditions.

GlpF, the glycerol facilitator in Escherichia coli, is responsible for uptake of water, glycerol and other small alditols (or linear polyalcohols) into the cell. ${ }^{19-21}$ It belongs to the aquaglyceroporin subfamily in the large aquaporin (AQP) protein family. ${ }^{22}$ Once they are translocated into the cytoplasm, glycerol molecules are phosphorylated and participate in the glycolytic pathway to generate energy. ${ }^{23}$ GlpF embedded in the membrane forms a homotetramer (Fig. 1a). Each monomer contains a right-handed bundle of six transmembrane $\alpha$-helices and two half-membrane-spanning $\alpha$-helices, which enclose an hourglass-shaped pore in each monomer (Fig. 1b). ${ }^{\mathbf{2 0 , 2 4}}$ The cytoplasmic and periplasmic ends of the pore entail two conically shaped vestibules, while the middle part is narrowed by two constrictive regions. The NPA region features two Asn-ProAla (NPA) motifs conserved among most AQPs, which form an electrostatic area excluding protons (Fig. 1b). ${ }^{25}$ The selectivity filter region (SF region, also named the ar/R region) forms the narrowest constriction of the pore with a radius of only $\sim 1.7 \AA$ (Fig. 1b). It is strongly amphipathic, lined by an arginine
(Arg206) and two aromatic amino acids (Trp48 and Phe200), which are important for substrate selectivity. ${ }^{\mathbf{2 0}, 26-28}$

Previous MD simulation studies of glycerol translocation through GlpF have employed various enhanced sampling methods to calculate the PMF profile, including steered $\mathrm{MD},{ }^{29}$ umbrella sampling, ${ }^{30}$ and adaptive biasing force (ABF) methods. ${ }^{31}$ All these simulations found the highest energy barrier in the SF region but with different heights $(4.0,3.2$, and $8.7 \mathrm{kcal} \mathrm{mol}^{-1}$ in ref. 29, 30, and 31, respectively). Besides the barrier in the SF region, the overall profiles of PMF from the three studies exhibit different features. The PMF calculated by the steered MD simulation demonstrates a rugged profile with a deep minimum at the periplasmic side, ${ }^{29}$ and this 'attractive vestibule' was suggested to be functionally important. ${ }^{29,32,33}$ On the other hand, the PMFs obtained by umbrella sampling and $\mathrm{ABF}$ calculations are smoother, and do not have the periplasmic 'attractive vestibule'. ${ }^{30,31}$ The kinetics of glycerol permeation have been estimated based on PMFs calculated by steered $\mathrm{MD}^{29,32}$ and $\mathrm{ABF}{ }^{31}$ giving quite different results. ${ }^{31,32}$ The discrepancies in both PMF estimation and kinetic analysis indicate the two challenging aspects of membrane transport simulation: sufficient sampling and proper construction of the kinetic model.

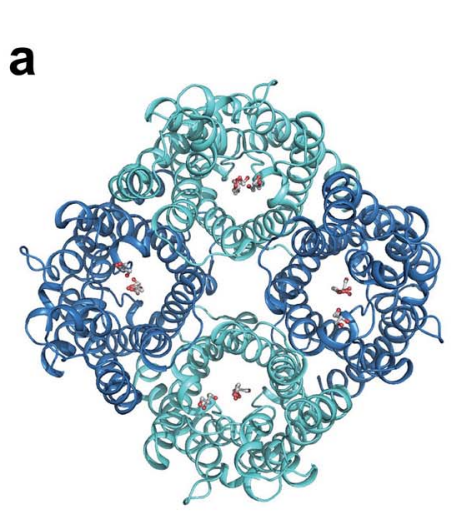

d

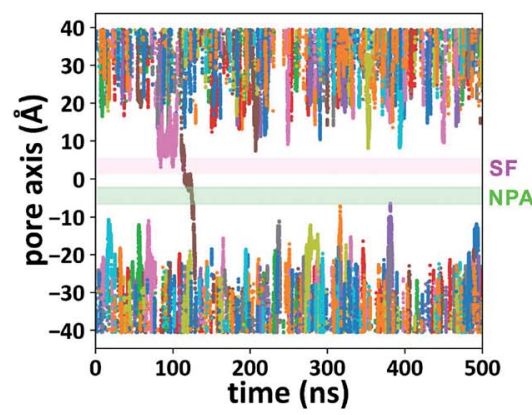

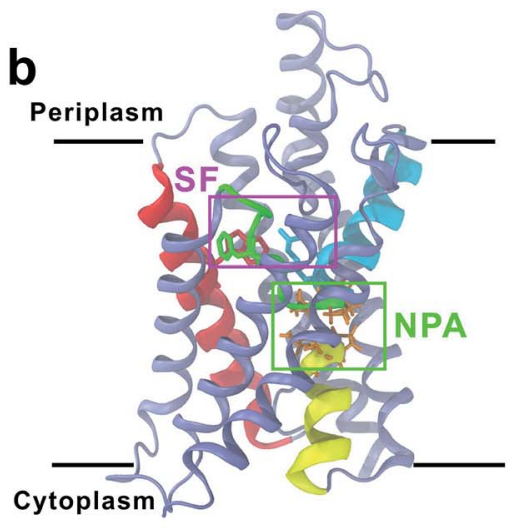

e

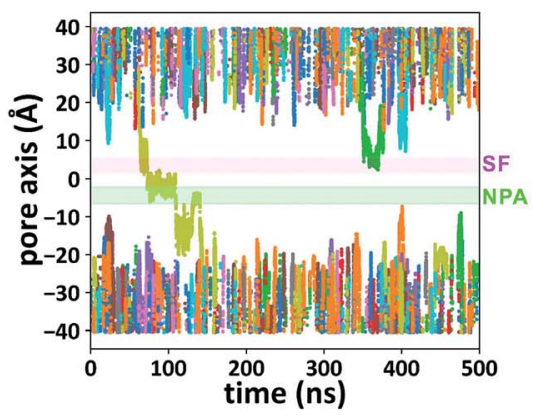

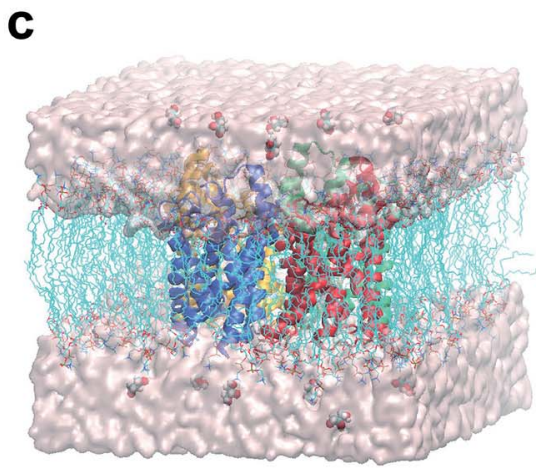

f

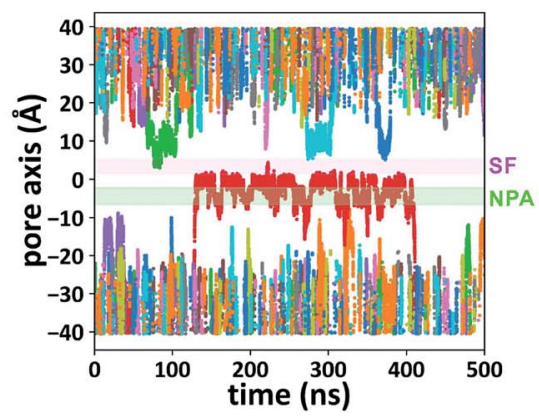

Fig. 1 Simulation of glycerol conduction through GlpF. (a) Top view of the crystal structure of the GlpF tetramer from the periplasmic side. (b) Atomic structure of one monomer of GlpF represented by cartoon mode. The three crucial residues (Trp48, Phe200, and Arg206) in the SF region are rendered by licorice mode and colored red, green, and cyan, respectively. The secondary structures they belong to are colored similarly. The two half helices (M3 and M7) are colored yellow and cyan, and the NPA motifs on them are rendered by licorice mode and colored orange. The NPA and SF regions are framed out by green and purple squares, respectively. Other parts of the protein are colored ice blue. (c) Simulation system of the GlpF tetramer embedded in a POPC lipid bilayer in the presence of $0.036 \mathrm{M}$ glycerol. (d-f) Three representative trajectories of the equilibrium MD simulations. All the trajectories of 12 glycerols in the simulation system are shown with different colors. The NPA and SF regions are highlighted by green and purple bars, respectively. 
Here, we performed extensive unbiased MD simulations of a GlpF system to achieve an adequate sampling of glycerol permeation. In addition to calculating the permeation PMF, we constructed a Markov state model (MSM) of glycerol transport and obtained all the rate constants of state transitions. Based on the MSM analysis, we built two stepwise kinetic models of glycerol translocation and solved the master equations under various boundary conditions, i.e. various glycerol concentrations at the two sides of the membrane. The calculated glycerol transport flux is in reasonable agreement with the experimental measurement at a certain extracellular glycerol concentration. More importantly, we achieved a holistic view of the glycerol transport capacity of GlpF under various conditions.

\section{Methods}

\subsection{System setup}

The crystal structure of GlpF was obtained from the Protein Data Bank (PDB ID: 1FX8). Missing atoms of residue Arg257 were added using the PDB2PQR server. ${ }^{34} \mathrm{p} K_{\mathrm{a}}$ values were estimated by using PROPKA 3.0, ${ }^{35}$ and it turns out that all titratable residues are in their default protonation states at $\mathrm{pH}$ 7.0. The GlpF tetramer was inserted into a pre-equilibrated palmitoyloleoyl-phosphocholine (POPC) lipid bilayer containing 256 lipids by using the "shrinking" method. ${ }^{36}$ Twelve glycerol molecules were added to the system, initially placed $>10 \AA$ away from the protein. Water, $\mathrm{Na}^{+}$, and $\mathrm{Cl}^{-}$were added to solvate and neutralize the system, and to maintain a physiological salinity of $150 \mathrm{mM}$. The final system contains 104173 atoms including 240 lipids and 18763 water molecules (Fig. 1c).

\subsection{Simulation parameters and data analysis}

We used the GROMACS 5.0 software package ${ }^{37}$ to conduct MD simulations with the all-atom CHARMM 36 force field ${ }^{38}$ and the TIP3P water model. A steepest descent algorithm was used for energy minimization for 100000 steps and the system equilibration lasted $20 \mathrm{~ns}$ with position restraints on the heavy atoms of protein in the NPT ensemble. We used semi-isotropic coupling to keep the pressure at 1 bar using the Berendsen algorithm ${ }^{39}$ with a time constant of $1 \mathrm{ps}$, and used the V-rescale algorithm $^{40}$ with a time constant of $0.1 \mathrm{ps}$ to maintain a constant temperature of $310 \mathrm{~K} .45$ trajectories were produced without any restraints, and each lasted $500 \mathrm{~ns}$, leading to an aggregation time of $22.5 \mu \mathrm{s}$. All bonds were constrained by the LINCS algorithm, ${ }^{41}$ and a time step of $2 \mathrm{fs}$ was used. The cutoffs of electrostatic interactions and van der Waals interactions were both set to $1.2 \mathrm{~nm}$. Long-range electrostatic interactions were computed by using the particle mesh Ewald (PME) method. ${ }^{42}$ The trajectories were analyzed using MDAnalysis. ${ }^{43}$ The pore radius was evaluated with the HOLE program. ${ }^{44} \mathrm{VMD}^{45}$ was used for structure visualization.

\subsection{Potential of mean force (PMF) calculation}

PMF $G(z)$ of glycerol translocation along the pore axis of GlpF was calculated according to the formula

$$
G(z)=-k_{\mathrm{B}} T \ln \langle n(z)\rangle+C
$$

where $z$ is the displacement of the center of mass (COM) of glycerol relative to the COM of GlpF along the pore axis, $k_{\mathrm{B}}$ is the Boltzmann constant, $T$ is the temperature, and $C$ is a constant to shift the value of PMF to zero when $z$ falls in the bulk phase. The pore of each GlpF monomer was defined as a cylinder with a radius of $17 \AA$ and the cylinder is extended into the bulk solvent as well. To merge the glycerol number statistics of the four monomers, we set one of the monomers in the crystal structure as the reference and superimposed the monomers in trajectories one by one to the reference. Glycerol molecules within the cylinder of each monomer were moved together with the protein during superimposition. Then we counted the number of glycerols in the cylinder by dividing them into bins with a length of $0.25 \AA . n(z)$ is the average number of glycerol molecules in a bin beginning at $z$. The error bars of PMF were estimated by using the bootstrapping method. ${ }^{46}$

The state-dependent PMF of the conformational and prochiral state $i$ of glycerol, $G_{i}(z)$, was defined as

$$
G_{i}(z)=-k_{\mathrm{B}} T \ln \left\langle n_{i}(z)\right\rangle+C
$$

where $n_{i}(z)$ is the average number of state $i$ glycerols in the bin at $z$ per snapshot, and $C$ has the same value as in eqn (1). Because

$$
\langle n(z)\rangle=\sum_{i=1}^{S}\left\langle n_{i}(z)\right\rangle
$$

where $S=18$ (the total number of different conformational and prochiral states of glycerol that we defined), the relationship between the integral PMF $G(z)$ and the state-dependent PMFs $G_{i}(z)$ can be explicitly expressed as

$$
\mathrm{e}^{-\frac{G(z)}{k_{\mathrm{B}} T}}=\sum_{i=1}^{S} \mathrm{e}^{-\frac{G_{i}(z)}{k_{\mathrm{B}} T}}
$$

\subsection{Markov state model analysis}

PyEMMA software ${ }^{47}$ was used to construct the Markov state model (MSM) of glycerol translocation. First, we split all trajectories into individual glycerol trajectories, i.e. each original trajectory was split into 12 one-glycerol trajectories. To ensure that the glycerol molecule moves continuously inside the cylinder of each monomer, we made further modifications. Once the glycerol moves across the periodic boundary of the system or out of the cylinder, we assumed that this trajectory is terminated and the remaining part was considered as a new trajectory. In this way, each one-glycerol trajectory was further divided into short and continuous trajectory fragments. Trajectory fragments that lasted for more than 1 ns were selected for further analysis. We define the system states with the COM of glycerol at the cytoplasmic side $(z<-25.5)$ and periplasmic side $(z>24.5)$ as state 0 and state $N$, respectively. Other states with the COM of glycerol between $z=-25.5$ and $z$ $=24.5$ were named state 1 to $N-1$, with each spanning a length of $0.25 \AA$ A. By this definition, we got 202 microstates. Next, by projecting all the trajectories onto the 202 states, we 
constructed the count matrix $\operatorname{Cij}(\tau)$, whose elements correspond to the number of observed transitions of glycerol from state $i$ to state $j$ after a lag time $\tau$. From the count matrix, we used the Bayesian estimator ${ }^{\mathbf{4 8} 49}$ to obtain the transition probability matrix $T$. If the model is Markovian, the dynamics can be propagated to long time scale dynamics:

$$
P(n \Delta t)=[T(\Delta t)]^{n} P(0)
$$

The relaxation times, or implied timescales, $\tau_{k}$, are computed from the eigenvalues:

$$
\tau_{k}=-\frac{\tau}{\ln \mu_{k}(\tau)}
$$

where $\mu_{k}$ is the $k$ th eigenvalue (sorted from the largest to the smallest, and the eigenvalue that equals to 1 is not considered) of the transition matrix with the lag time $\tau$. In general, if the model is Markovian, the implied timescales plateau and become constant with the increase of lag time. We then applied the PCCA + algorithm ${ }^{50}$ to lump all the microstates into 8 macrostates.

\subsection{Transition path theory analysis}

To estimate the rates of glycerol transport through the pore, we used transition path theory (TPT). ${ }^{51-53}$ In TPT, two sets of states, source states $A$ and sink states $B$, are defined to specify the transition from $A$ to $B$. All remaining states are considered to be intermediate states. Then, the forward-committor probability $q_{i}^{+}$, defined as the probability that the system, when being in state $i$, will reach state $B$ next rather than state $A$, can be computed from the transition matrix. Similarly, the backwardcommittor probability $q_{i}{ }^{-}$is calculated as the probability that the system, being in state $i$, was previously in state $A$ rather than in state $B$. The total flux $F$ from $A$ to $B$ per unit time $\tau$ is calculated using the relationship

$$
F=\sum_{i \in A} \sum_{j \notin A} \pi_{i} T_{i j} q_{j}^{+}
$$

where $\pi_{i}$ is the stationary population of state $i$ and $T_{i j}$ is the element of the transition matrix specifying the transition probability from state $i$ to state $j$. The rate constant of the transition from $A$ to $B$ can be calculated as

$$
k_{A B}=\frac{F}{\tau \sum_{i=1}^{m} \pi_{i} q_{i}^{-}}
$$

where the summation runs over all states in the model. Here, we calculated the transition rate constants between the eight macrostates based on the 202-microstate MSM. It is worth noting that the step of glycerol entering the channel from the bulk solvent (either the periplasm or cytoplasm) is a second order reaction, in which glycerol binds to the protein. However, if the glycerol concentration remains constant at both sides of the membrane, this step could be viewed as a pseudo-first order reaction. The rate constants derived from the MSM correspond to pseudo-first order rate constants at a glycerol concentration of $0.036 \mathrm{M}$. Therefore, we give the second-order rate constants $\left(k_{01}\right.$ and $\left.k_{76}\right)$ in Fig. 4 and Table $\mathrm{S} 2 \uparrow$ by dividing them by $0.036 \mathrm{M}$.

\section{Results and discussion}

\subsection{Equilibrium MD simulations of spontaneous glycerol permeation}

Forty-five $500 \mathrm{~ns}$ unbiased all-atom MD simulations (total simulation time of $22.5 \mu \mathrm{s}$ ) were performed for the membraneembedded GlpF tetramer with a glycerol concentration of $0.036 \mathrm{M}$ at both sides of the lipid bilayer (Fig. 1c). We have observed 24 times the spontaneous permeation of glycerol through the entire pore of GlpF, among which 15 involved translocations from the periplasmic side to the cytoplasmic side $(\mathrm{P} \rightarrow \mathrm{C})$ and the other 9 times the translocations were in the opposite direction $(\mathrm{C} \rightarrow \mathrm{P})$. In addition, another 15 times glycerol passage through the narrowest SF region was observed, but in these cases, glycerol diffused back and did not permeate to the other side of the membrane. Inspection of the trajectories reveals several interesting points. First, in the 24 spontaneous permeations of glycerol, the average time a conducting glycerol molecule spends inside the channel is short $(40 \pm 25 \mathrm{~ns}$, Table S1 $\dagger$ ) relative to the total simulation time. For a majority of the simulation time, glycerols were in the bulk solution or bound somewhere inside the channel, while the successful permeations were fleeting (Fig. 1d-f). In this scenario, glycerol translocations are barrier-crossing rare events. Second, there exist several sites inside the channel where glycerol has relatively long residence time (Fig. 1f), indicating that there are several stable states during the translocation process. Third, at the glycerol concentration used in our simulation, it is more probable that there is only one glycerol molecule inside the channel during translocation. The probability of finding one glycerol inside the channel is $24.1 \%$ with respect to the total simulation time, while the probability of finding two or more glycerol molecules simultaneously inside the same channel is $3.7 \%$. This implies that we may ignore the interaction between glycerol molecules during translocation.

\subsection{Potential of mean force (PMF) for glycerol translocation}

To obtain a quantitative thermodynamic description of glycerol transport, we calculated the 1-dimensional PMF of glycerol translocation along the pore axis ( $z$ axis) from the MD trajectories (Fig. 2a and b, see the Methods section). As expected, the highest energy barrier $\left(3.1 \pm 0.1 \mathrm{kcal} \mathrm{mol}^{-1}\right)$ in the PMF profile is located in the SF region (Fig. $2 \mathrm{~b}, z=2.0 \AA$ ). This is generally in agreement with the previous PMF calculations. ${ }^{29-31}$ The PMF estimation using umbrella sampling gave an SF barrier of $3.2 \mathrm{kcal} \mathrm{mol}{ }^{-1}$, which is very close to our result. ${ }^{30}$ However, differences are evident when our result is compared with those derived from the steered $\mathrm{MD}^{29}$ and $\mathrm{ABF}$ methods, ${ }^{31}$ which gave higher barriers (4.0 and $8.7 \mathrm{kcal} \mathrm{mol}^{-1}$ ) and different shapes at several places. For example, the PMF from the steered MD simulation gave a deep energy well at the periplasmic vestibule, ${ }^{29}$ but our simulation and the other two studies ${ }^{30,31}$ did not find this feature (Fig. 2b). In the crystal structure of GlpF, three glycerol molecules were trapped inside the channel, ${ }^{20}$ the 

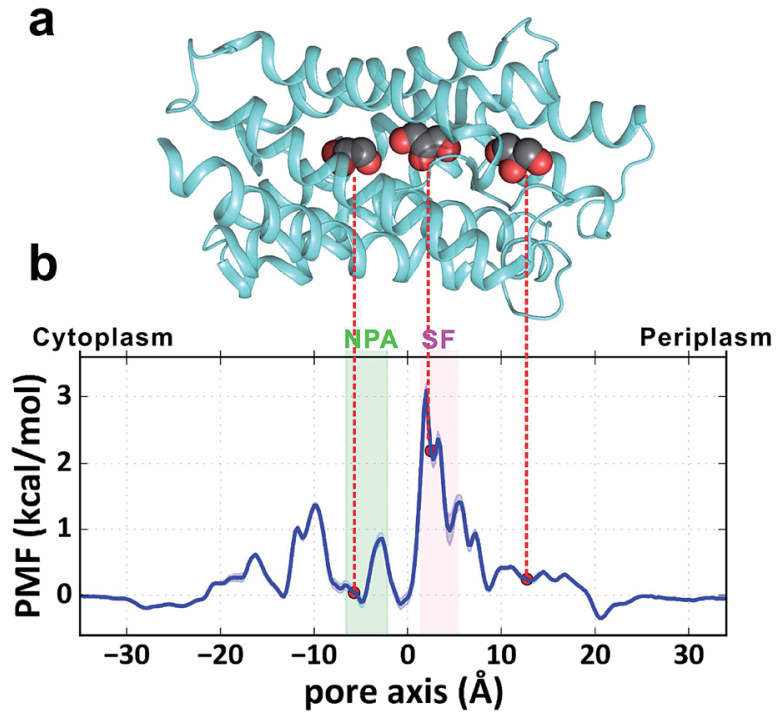

Fig. 2 PMF of glycerol transport through the GlpF channel. (a) Crystal structure of one monomer of GlpF. The three cocrystallized glycerol molecules are shown in a sphere model. (b) 1D PMF of glycerol transport along the pore axis. The zero point of the pore axis is at the center of mass of GlpF and the zero point of free energy is set when glycerol is in the bulk solvent. The positions corresponding to the bound glycerols in the crystal structure are indicated by red circles.

positions of which all correspond to local minima in our PMF profile (Fig. 2a and b). The second glycerol sits near the SF region with relatively high free energy (Fig. 2). This 'unfavorable' location of glycerol is most likely due to the high glycerol concentration in the crystallographic experiment $(\sim 1.6 \mathrm{M})$. In general, our PMF profile is more similar to the PMF calculated by umbrella sampling. ${ }^{30}$ It is worth noting that our simulation time is much longer and there is no bias in our simulation. The convergence of our PMF calculation was also verified (Fig. S1 $†$ ). Another notable difference between our simulation and the previous studies is that our simulation system contains twelve glycerol molecules and there is a probability for multiple glycerols appearing inside the channel, while single glycerol translocation was forced in the other simulations.

\subsection{Stereoselectivity of the SF region of GlpF for glycerol permeation}

Both the crystal structure and the PMF profile suggest that the motion of glycerol is highly restrained as it passes through the $\mathrm{SF}$ region. To analyze the stereoselectivity of the SF region, we used three Euler angles $(\varphi, \theta$, and $\psi)$ to characterize the orientation of glycerol with respect to the protein (Fig. 3a). The reference state with all three angles being zero is defined as the most probable orientation of glycerol in the SF region. In bulk solution, the three angles $(\theta, \varphi$, and $\psi)$ exhibit even or cosine distributions (Fig. 3b, black curves), indicating random orientation. In the SF region, the three Euler angles are highly restricted around $0^{\circ}$ or $\pm 180^{\circ}$ (Fig. 3b, red curves). Accordingly, two orientational or prochiral states of glycerol can be defined as $\mathrm{C}_{1}\left(\varphi \approx \theta \approx \psi \approx 0^{\circ}\right)$ and $\mathrm{C}_{3}\left(\varphi \approx 180^{\circ}, \theta \approx \psi \approx 0^{\circ}\right)$, with the $\mathrm{C}_{1}$ or $\mathrm{C}_{3}$ atom of glycerol (see Fig. 3a for atom numbering) a

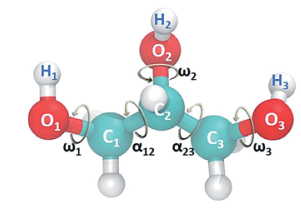

C

b
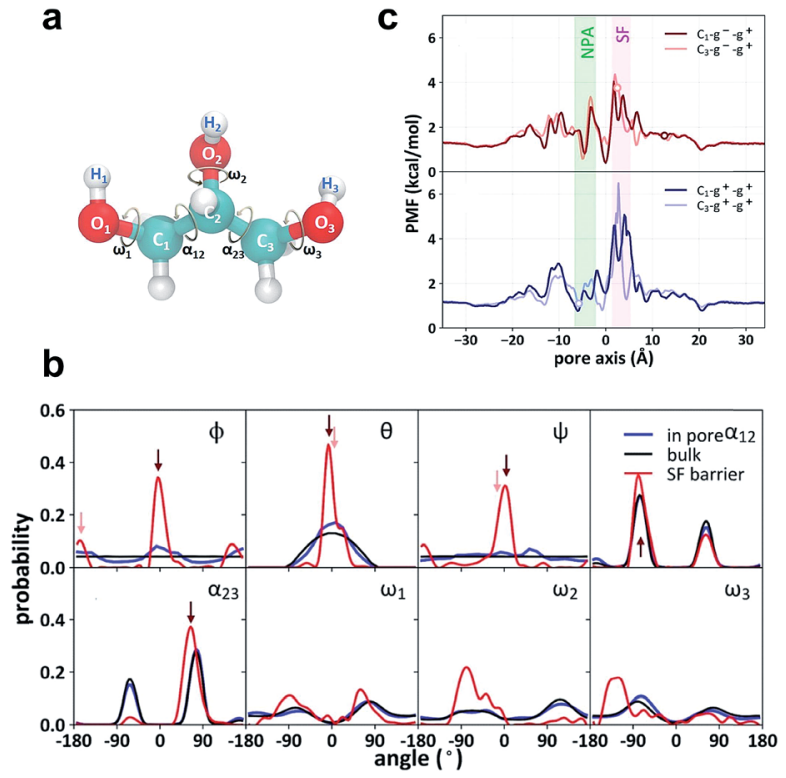

Fig. 3 Stereoselectivity of the SF region for glycerol. (a) Structure of glycerol and the definition of the torsion angles $\alpha_{12}, \alpha_{23}, \omega_{1}, \omega_{2}$, and $\omega_{3}$. (b) Distributions of the Euler angles $\phi, \theta$, and $\psi$, backbone torsion angles $\alpha_{12}$ and $\alpha_{23}$ and the side chain torsion angles $\omega_{1}, \omega_{2}$, and $\omega_{3}$. The Euler angles denote sequential rotations of glycerol around the $x^{-}, y^{\prime}-$, and $z^{\prime \prime}$-axis to overlap with one representative structure of the $C_{1}-g^{-}-$ $g^{+}$state. The angles corresponding to $\mathrm{C}_{1}-g^{-}-g^{+}$and $\mathrm{C}_{3}-g^{-}-g^{+}$states are indicated by dark red and light red arrows, respectively. (c) Statedependent PMFs of the $\mathrm{C}_{1}-g^{-}-g^{+}$, the $\mathrm{C}_{3}-g^{-}-g^{+}$, the $\mathrm{C}_{1}-g^{+}-g^{+}$and the $\mathrm{C}_{3}-g^{+}-g^{+}$states along the pore axis. The zero points of the statedependent PMFs were set according to that of the integral PMF to explicitly account for the confinement penalty from isolating the selected conformational and prochiral state from the state mixture in the bulk phase. The three glycerol molecules co-crystallized with GlpF are in the $\mathrm{C}_{1}-g^{-}-g^{+}, \mathrm{C}_{3}-g^{-}-g^{+}$and $\mathrm{C}_{3}-g^{+}-g^{+}$states, respectively. Their positions are indicated by circles in the respective PMF curves.

entering the SF constriction first from the periplasmic side, respectively. On the other hand, glycerol has intramolecular conformational flexibility. To characterize the conformational selectivity, we examined the two backbone torsion angles $\alpha_{12}$ $\left(\mathrm{O}_{1}-\mathrm{C}_{1}-\mathrm{C}_{2}-\mathrm{O}_{2}\right)$ and $\alpha_{23}\left(\mathrm{O}_{3}-\mathrm{C}_{3}-\mathrm{C}_{2}-\mathrm{O}_{2}\right)$, and the three hydroxylinvolving torsion angles $\omega_{1}, \omega_{2}$, and $\omega_{3}$ of glycerol (Fig. 3a). The distributions of the five torsion angles in the SF region are different from those in the bulk phase (Fig. $3 \mathrm{~b}$, red curves $v s$. black curves). Torsion angles $\omega_{1}, \omega_{2}$, and $\omega_{3}$ display broad and even distributions in solution and in the pore (Fig. $3 \mathrm{~b}$ ). The SF region slightly restricts the rotation of the $\mathrm{C}-\mathrm{O}$ bonds, leading to narrowed distributions (Fig. 3b). The backbone torsion angles $\alpha_{12}$ and $\alpha_{23}$ distribute mainly around $75^{\circ}$ and $-75^{\circ}$, corresponding to the so-called gauche $e^{+}$or $g^{+}\left(0-120^{\circ}\right)$ and gauche $e^{-}$or $g^{-}\left(-120-0^{\circ}\right)$ conformations, respectively (Fig. $\left.3 \mathrm{~b}\right)$. The anti or $a\left(-180\right.$ to $-120^{\circ}$ or $\left.120-180^{\circ}\right)$ conformation, however, is much less probable (Fig. $3 \mathrm{~b}$ ). For simplicity, we only use the backbone torsion angles $\alpha_{12}$ and $\alpha_{23}$ to categorize the conformational states of glycerol, and the combinations of the three states $\left(g^{+}, g^{-}\right.$and $\left.a\right)$ of the two torsion angles give nine conformational states $\left(g^{+}-g^{+}, g^{-}-g^{+}, g^{+}-g^{-} \ldots\right)$. By combining the two prochiral $\left(\mathrm{C}_{1}\right.$ and $\left.\mathrm{C}_{3}\right)$ and nine conformational states, we 
define eighteen overall states of a glycerol molecule, named $\mathrm{C}_{1}$ $g^{-}-g^{+}, \mathrm{C}_{3}-\mathrm{g}^{-}-\mathrm{g}^{+}$, etc.

The definition of the prochiral-conformational state of glycerol enables us to decompose the PMF of glycerol translocation (Fig. 2b) into 18 state-dependent PMFs (Fig. 3c and S2, $\dagger$ see the Methods section for details). These state-dependent PMFs provide a comprehensive picture of the selectivity of the SF for glycerol permeation. The PMF of $\mathrm{C}_{1}-g^{-}-g^{+}$has the lowest energy barrier in the $\mathrm{SF}$ region (Fig. 3c), where this state occupies $52.8 \%$ of the total population of the permeable glycerols. The $\mathrm{C}_{3}-g^{-}-g^{+}$state is less permeable with a population of $13.7 \%$ in the SF region and a slightly higher energy barrier (Fig. 3c). The $g^{+}-g^{+}$conformation is less permeable and forms fewer hydrogen bonds with the SF region than the $g^{-}-g^{+}$one. Therefore, the PMFs of $\mathrm{C}_{1}-g^{+}-g^{+}$and $\mathrm{C}_{3}-g^{+}-g^{+}$have higher energy barriers in the $\mathrm{SF}$ region (Fig. 3c). The other states, such as $\mathrm{C}_{1}-g^{-}-g^{-}, \mathrm{C}_{3}-g^{-}-g^{-}, \mathrm{C}_{1}-g^{+}-g^{-}$, and $\mathrm{C}_{3}-g^{+}-g^{-}$, are totally impermeable during the simulation (Fig. $\mathrm{S} 2 \dagger$ ). This result generally agrees with the crystal structure and the previous simulation studies. ${ }^{29,31}$ In the crystal structure, the glycerol near the $\mathrm{SF}$ region adopts the $\mathrm{C}_{3}-g^{--}-g^{+}$state.

In line with the stereoselectivity of the SF region shown in the state-dependent PMFs, the probability of transitions among different prochiral-conformational states of glycerol is extremely low in the SF region (Fig. S3†). We examined all 39 passages through the $\mathrm{SF}$ region by tracing the prochiralconformational state evolution of glycerol (Fig. S4†). It was found that 14 times glycerol passed through the SF region in the $\mathrm{C}_{1}-g^{-}-g^{+}$state without transitions (Fig. S4a $\dagger$ ), and for another 4 passages the $\mathrm{C}_{1}-g^{-}-g^{+}$state was predominant with only one or two transitions (Fig. S4a $\dagger$ ). The $\mathrm{C}_{3}-g^{-}-g^{+}$state is similarly favorable by being exclusive in 8 of the passages and being predominant in another 8 (Fig. S4b $\dagger$ ). The $g^{+}-g^{+}$conformer, however, always experienced transitions during the passages (Fig. S4c $\dagger$ ). Overall, the state-dependent PMFs and the state transition analysis all indicate that the SF region has high stereoselectivity for glycerol. Nevertheless, it is notable that the non-polarizable force field employed in this study may limit the accuracy of a detailed description of the conformational motion of glycerol in a highly complicated environment.

\subsection{Markov state model analysis of the glycerol transport process}

The 1-D PMF of glycerol permeation reveals several local energy minima along the pore axis (Fig. 2b), entailing translocation intermediate states. To obtain kinetic information on glycerol transport, we performed Markov state model (MSM) analysis of the MD simulation trajectories. First, we divided the channel along the pore axis (running from $z=-25.5 \AA$ to $24.5 \AA$ ) into 200 bins, each of which spans $0.25 \AA$. The microstates of the system are defined by the locations of the glycerol molecule in these bins. Therefore, snapshots with the COM of glycerol at the same bin belong to the same microstate. For the snapshots with no glycerol inside the channel, two microstates are defined with glycerol in the periplasmic and cytoplasmic side bulk solutions, respectively. The 202 microstates are further lumped into eight macrostates (see the Methods section for more details). The implied timescales for both 202microstate and 8-macrostate MSMs reach the plateau when the lag time is more than $80 \mathrm{ps}$, indicating that the model is Markovian (Fig. S5a and c $\dagger$ ), and we further validated the MSM using the Chapman-Kolmogorov test ${ }^{54}$ (Fig. S5b and $d \dagger$ ). The 1-D PMF of glycerol translocation calculated based on the MSM agrees well with that calculated using raw MD trajectories (Fig. 4). Macrostates were numbered 0 to 7 from the periplasm side to the cytoplasm side dictating the positions of glycerol. States 1 to 6 represent glycerol locations inside the channel (Fig. 4). State 0 and state 7 , the states with glycerol in the periplasm and cytoplasm respectively, have a total equilibrium population of $\sim 55 \%$ (Fig. 4). Among the six states with glycerol inside the pore, state 1 has a relatively large probability of $11.04 \%$. This corresponds to the periplasmic vestibule. A previous simulation study found that this area is the global minimum in the PMF profile, ${ }^{55}$ and a glycerol molecule is trapped here in the crystal structure. ${ }^{20}$ State 6 , the state near the cytoplasmic entrance, has a comparable population $(12.5 \%)$ to state 1 . Therefore, the asymmetry of GlpF in our calculation is not as significant as shown in the previous simulation. ${ }^{55}$ The rate constants of all transitions between two adjacent states were calculated using the TPT ${ }^{51-53}$ from the 202microstate MSM (Fig. 4 and Table S2, $\uparrow$ see the Methods section for more details). In line with the PMF profile, the slowest step during permeation is crossing the SF barrier. It is worth noting that the detailed balance conditions are satisfied in the rate constant estimations, ${ }^{\mathbf{4 7}, 56}$ ensuring the reliability of the following kinetic analysis using these rate constants.

\subsection{Glycerol permeation flux under various concentration gradients}

The above MD simulations were performed under equilibrium conditions, which assume equal glycerol concentration $(0.036$ $\mathrm{M})$ in the periplasm and the cytoplasm. This is quite different from the in vivo situation in $E$. coli, where there is a glycerol

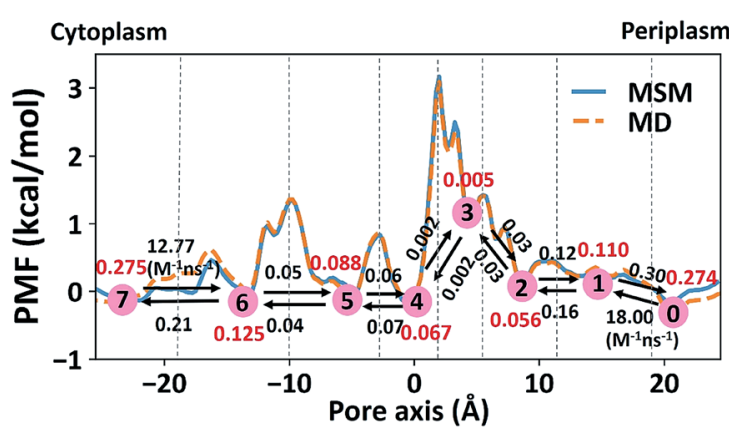

Fig. 4 Markov state model analysis of glycerol transport through GlpF. Eight macrostates (state 0 to 7) were constructed from the MSM and are labeled in red circles and the corresponding populations are in red. Dashed lines represent the boundaries between different states, lying at $-19,-10,-3,2.25,5,11.5$, and $19 \AA$, respectively. The values near the black arrows are rate constants of the state transitions with units of $\mathrm{ns}^{-1}$ if not specified. 
concentration difference across the inner membrane. Glycerol molecules are phosphorylated by glycerol kinase once they are translocated into the cytoplasm; therefore, the cytoplasmic glycerol concentration is supposed to remain at a low level. To calculate the glycerol transport flux under various concentration gradients, we employed two kinetic models, under the assumption that the rate constants derived from the equilibrium MD simulation are still valid at non-equilibrium conditions. In the first model, only one glycerol molecule is allowed to appear in the channel during translocation, and a 7-state kinetic model based on the MSM was constructed (Fig. 5a). Here, different from the MSM, states 0 and 7 merge into one state (state 0), which represents the state with no glycerol inside the channel. The master equations of this kinetic model are as follows:

$$
\begin{gathered}
\frac{\mathrm{d}}{\mathrm{d} t} P_{0}=-P_{0} k_{01} c_{\mathrm{out}}+P_{1} k_{10}-P_{0} k_{06} c_{\mathrm{in}}+P_{6} k_{60} \\
\frac{\mathrm{d}}{\mathrm{d} t} P_{1}=-P_{1} k_{12}-P_{1} k_{10}+P_{0} k_{01} c_{\mathrm{out}}+P_{2} k_{21}
\end{gathered}
$$

$$
\frac{\mathrm{d}}{\mathrm{d} t} P_{i}=-P_{i} k_{i, i+1}-P_{i} k_{i, i-1}+P_{i-1} k_{i-1, i}+P_{i+1} k_{i+1, i} \quad i=2,3,4,5
$$

$$
\begin{gathered}
\frac{\mathrm{d}}{\mathrm{d} t} P_{6}=-P_{6} k_{60}-P_{6} k_{65}+P_{5} k_{56}+P_{0} k_{06} c_{\text {in }} \\
\sum_{i=0}^{6} P_{i}=1
\end{gathered}
$$

where $P_{1}-P_{6}$ are probabilities of the six states with one glycerol in the channel, $P_{0}$ is the probability of state $0, k_{i j}$ is the rate constant for the transition from state $i$ to state $j$, and $c_{\text {out }}$ and $c_{\text {in }}$ are constant concentrations of glycerol in the periplasm and cytoplasm, respectively. The values of the rate constant $\left(k_{i j}\right)$ are obtained from the above MSM analysis (Fig. 4 and Table S2 $\dagger$ ). Note that $k_{06}$ and $k_{60}$ correspond to $k_{76}$ and $k_{67}$ in the MSM, respectively. The master equations were solved numerically as time evolved till all the state probabilities remained unchanged, indicating the achievement of a steady state. The net flux of
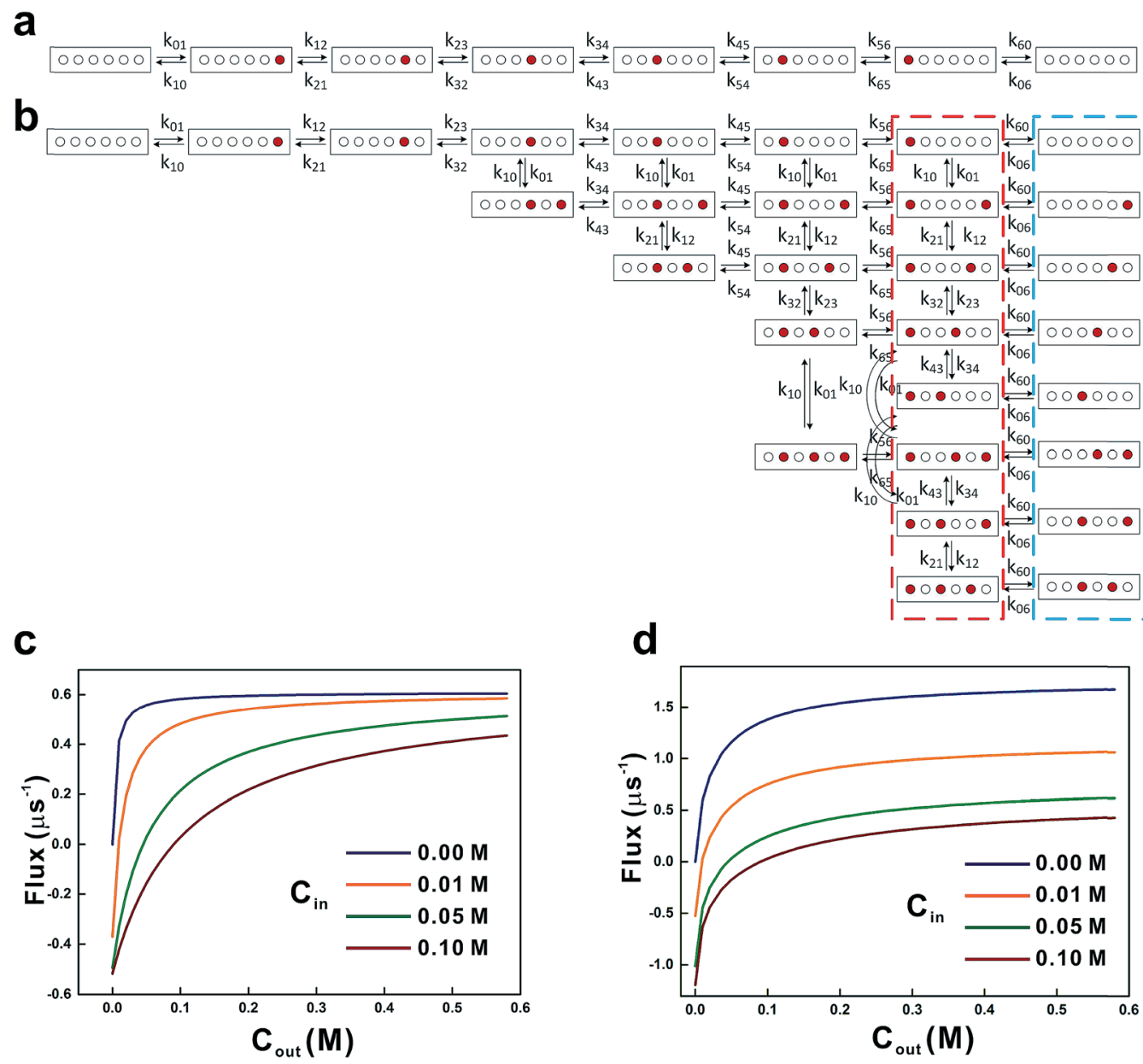

Fig. 5 Kinetic models for glycerol transport. The 7-state kinetic model (a) and the 21-state kinetic model (b) were used to calculate the glycerol influx rates. Red and open circles represent the glycerol occupied and the vacant sites, respectively. Transition rate constants between various states are indicated near the arrow. All the states encircled by the red and blue dashed lines (with probabilities $P_{\mathrm{r}}$ and $P_{\mathrm{b}}$, respectively) contribute to the net influx $J_{\overrightarrow{P C}}$ in eqn (16). Net influxes of glycerol at various periplasmic $\left(C_{\text {out }}\right)$ and cytoplasmic $\left(C_{\text {in }}\right)$ glycerol concentrations were calculated using the 7-state kinetic model (c) and the 21-state kinetic model (d). 
glycerol from the periplasm to the cytoplasm $J_{\mathrm{P} \rightarrow \mathrm{C}}$ can be calculated as

$$
J_{\mathrm{P} \rightarrow \mathrm{C}}=P_{6} k_{60}-P_{0} k_{06} c_{\text {in }}
$$

In the second model, the channel is allowed to accommodate more than one glycerol molecule. Considering the size of glycerol $(\sim 5 \AA)$, we assume that the adjacent sites inside the pore cannot accommodate glycerols at the same time. Consequently, there are totally 21 possible states with at most three glycerols inside the channel (Fig. 5b). If we further assume that only one glycerol moves in all the state transitions (referred to as the 'one-glycerolhopping' model), a 21-state kinetic model is thus established (Fig. 5b). The corresponding master equation reads as follows:

$$
\frac{\mathrm{d}}{\mathrm{d} t} P_{i}=-\sum_{m} P_{i} k_{i m}^{\prime}+\sum_{n} P_{n} k_{n i}^{\prime}
$$

where $P_{i}$ is the probability of state $i, m$ denotes all the states into which state $i$ could transform through one-glycerol-hopping, $n$ denotes all the states that could transform into state $i$ through one-glycerol-hopping, and $i=1,2, \ldots, 21 . k_{i m}^{\prime}$ and $k_{n i}^{\prime}$ are the rate constants for transition among the different states. Note that these rate constants can also be derived from the MSM since we assume the one-glycerol-hopping model (see Fig. 5b for more details). The steady-state net flux $J_{\mathrm{P} \rightarrow \mathrm{C}}$ in this kinetic model can be calculated as

$$
J_{\mathrm{P} \rightarrow \mathrm{C}}=\sum_{\mathrm{r}} P_{\mathrm{r}} k_{60}-\sum_{\mathrm{b}} P_{\mathrm{b}} k_{06} c_{\text {in }}
$$

where $P_{\mathrm{r}}$ and $P_{\mathrm{b}}$ refer to the probabilities of the states in the red and blue dotted boxes (Fig. 5b), respectively.

By solving the master equations of the two above-mentioned kinetic models at $c_{\text {in }}$ and $c_{\text {out }}$, we obtained glycerol transport flux rates under various non-equilibrium conditions (Fig. $5 \mathrm{c}$ and d). Although glycerol is phosphorylated in the cytoplasm, the cytoplasmic glycerol concentration is unnecessarily zero. Here, we chose four values of $c_{\text {in }}(0 \mathrm{M}, 0.01 \mathrm{M}, 0.05 \mathrm{M}$, and 0.1 $\mathrm{M})$ to calculate the steady-state influx rate of glycerol while varying the $c_{\text {out }}$ in the range of $0-0.6 \mathrm{M}$. In the first model, with the increase of $c_{\text {out }}$ the net flux $J_{\overrightarrow{\mathrm{PC}}}$ saturates very quickly at low cytoplasmic concentrations ( $c_{\text {in }}=0 \mathrm{M}$ and $0.01 \mathrm{M}$, Fig. $5 \mathrm{c}$ ). The saturated flux rate is $\sim 0.6 \mu \mathrm{s}^{-1}$. In the second model where the channel may accommodate multiple glycerol molecules, the net fluxes are generally larger and saturate slowly (Fig. $5 \mathrm{~d}$ ). At zero cytoplasmic glycerol concentration, the saturated flux rate is $\sim 1.6 \mu \mathrm{s}^{-1}$ (Fig. 5d). Experimentally, the glycerol flux rate was estimated to be $0.2 \mu \mathrm{s}^{-1}$ based on measurement at a $c_{\text {out }}$ of $0.5 \mathrm{M},{ }^{57}$ while $c_{\text {in }}$ is unknown. In our two models, when $c_{\text {out }}=$ $0.5 \mathrm{M}$, the influx rates range from 0.4 to $0.6 \mu \mathrm{s}^{-1}$ (model 1) and 0.4 to $1.6 \mu \mathrm{s}^{-1}$ (model 2), respectively (Fig. $5 \mathrm{c}$ and d). It is worth noting that the experimental estimation has a large uncertainty. For example, it was assumed that the copy number of GlpF in $E$. coli equals to that of glycerol kinase, and the copy number of glycerol kinase $\left(6 \times 10^{3}\right.$ molecules per cell) is estimated based on enzyme activity. A recent proteomic study using a mass spectrometer, however, gave a quite different estimation $(1.1 \times$
$10^{3}$ molecules per cell), which implies a higher glycerol flux rate. Although the second model seemingly overestimates the influx rate of glycerol, it is probably the more reasonable model. The glycerol concentration in our equilibrium simulation is quite low $(0.036 \mathrm{M})$; therefore, under the non-equilibrium conditions close to the experimental situation, GlpF is very likely to accommodate more glycerols than observed in the above MD simulation. To test this hypothesis, we performed additional simulations at several higher glycerol concentrations of $0.1 \mathrm{M}$, $0.15 \mathrm{M}, 0.2 \mathrm{M}$, and $0.25 \mathrm{M}$, respectively. Five $50 \mathrm{~ns}$ long simulations were conducted at each concentration. It turns out that with the increase of glycerol concentration, it is possible that more binding sites in the channel are occupied simultaneously (Fig. S6 $\dagger$ ). At higher concentrations, there is a small probability of accommodating more than three glycerols in the channel ( $\sim 3 \%$ under $0.25 \mathrm{M}$ ) (Fig. S6 $\dagger$ ). It should be noted that the simulation is conducted under equilibrium conditions, and there should be fewer glycerols in the channel under nonequilibrium conditions with concentration gradients. Therefore, the 21-state model that allows at most three glycerols in the channel is a good approximation.

\section{Conclusion}

In this work, we conducted large scale equilibrium MD simulations of glycerol translocation through GlpF. Tens of times spontaneous glycerol permeation was observed. The PMF profile of glycerol transport confirms the functional importance of the SF region, and the stereoselectivity analysis shows that the $\mathrm{C}_{3}-g^{-}-g^{+}$ state of glycerol has the highest permeability. By performing MSM analysis, we obtained the rate constants of state transitions during glycerol transport, based on which we were able to build kinetic models for glycerol influx under non-equilibrium conditions. The calculated net fluxes under various glycerol concentration gradients using the two kinetic models provide a holistic view of the glycerol transport capacity of GlpF. Our work demonstrates that long-timescale equilibrium simulation combined with MSM analysis could achieve the ' $a b$ initio' calculation of kinetics and complement the structural and physiological experiments, providing a detailed understanding of channel transport.

\section{Conflicts of interest}

There are no conflicts to declare.

\section{Acknowledgements}

This work was supported by the Ministry of Science and Technology of the People's Republic of China (No. 2016YFA0501702), National Natural Science Foundation of China (21473034, $21773038,21877017)$. This research made use of the resources of the computer clusters at the computer center at Fudan University.

\section{Notes and references}

1 C. Maffeo, S. Bhattacharya, J. Yoo, D. Wells and A. Aksimentiev, Chem. Rev., 2012, 112, 6250-6284. 
2 J. Weng and W. Wang, Adv. Exp. Med. Biol., 2014, 805, 305-329. 3 C. Kutzner, H. Grubmuller, B. L. de Groot and U. Zachariae, Biophys. J., 2011, 101, 809-817.

4 C. Kutzner, D. A. Kopfer, J. P. Machtens, B. L. de Groot, C. Song and U. Zachariae, Biochim. Biophys. Acta, Biomembr., 2016, 1858, 1741-1752.

5 F. Noe, I. Horenko, C. Schutte and J. C. Smith, J. Chem. Phys., 2007, 126, 155102.

6 C. Schütte, A. Fischer, W. Huisinga and P. Deuflhard, J. Comput. Phys., 1999, 151, 146-168.

7 W. C. Swope, J. W. Pitera and F. Suits, J. Phys. Chem. B, 2004, 108, 6571-6581.

8 J. H. Prinz, H. Wu, M. Sarich, B. Keller, M. Senne, M. Held, J. D. Chodera, C. Schutte and F. Noe, J. Chem. Phys., 2011, 134. 9 R. Zwanzig, J. Stat. Phys., 1983, 30, 255-262.

10 A. C. Pan and B. Roux, J. Chem. Phys., 2008, 129, 064107.

11 V. Schultheis, T. Hirschberger, H. Carstens and P. Tavan, J. Chem. Theory Comput., 2005, 1, 515-526.

12 N. Singhal, C. D. Snow and V. S. Pande, J. Chem. Phys., 2004, 121, 415-425.

13 N. V. Buchete and G. Hummer, J. Phys. Chem. B, 2008, 112, 6057-6069.

14 W. Zheng, M. Andrec, E. Gallicchio and R. M. Levy, J. Phys. Chem. B, 2009, 113, 11702-11709.

15 V. A. Voelz, G. R. Bowman, K. Beauchamp and V. S. Pande, J. Am. Chem. Soc., 2010, 132, 1526-1528.

16 Y. Feng, L. Zhang, S. Wu, Z. Liu, X. Gao, X. Zhang, M. Liu, J. Liu, X. Huang and W. Wang, Angew. Chem., Int. Ed. Engl., 2016, 55, 13990-13994.

17 D. Wang, J. Weng and W. Wang, J. Comput. Chem., 2019, 40, 1440-1448.

18 J. Weng and W. Wang, J. Chem. Inf. Model., 2019, 59, 23592366.

19 H. Tsukaguchi, C. Shayakul, U. V. Berger, B. Mackenzie, S. Devidas, W. B. Guggino, A. N. Van Hoek and M. A. Hediger, J. Biol. Chem., 1998, 273, 24737-24743.

20 D. Fu, A. Libson, L. J. Miercke, C. Weitzman, P. Nollert, J. Krucinski and R. M. Stroud, Science, 2000, 290, 481-486.

21 C. Maurel, J. Reizer, J. I. Schroeder, M. J. Chrispeels and M. H. Saier, J. Biol. Chem., 1994, 269, 11869-11872.

22 G. M. Preston, T. P. Carroll, W. B. Guggino and P. Agre, Science, 1992, 256, 385-387.

23 E. C. Lin, Escherichia coli and Salmonella: cellular and molecular biology, ASM Press, Washington, DC, 2nd edn, 1996, pp. 307-342.

24 E. Tajkhorshid, P. Nollert, M. O. Jensen, L. J. Miercke, J. O'Connell, R. M. Stroud and K. Schulten, Science, 2002, 296, 525-530.

25 B. L. de Groot and H. Grubmuller, Science, 2001, 294, 2353-2357.

26 M. J. Borgnia, D. Kozono, G. Calamita, P. C. Maloney and P. Agre, J. Mol. Biol., 1999, 291, 1169-1179.

27 E. Beitz, B. Wu, L. M. Holm, J. E. Schultz and T. Zeuthen, Proc. Natl. Acad. Sci. U. S. A., 2006, 103, 269-274.

28 D. F. Savage, P. F. Egea, Y. Robles-Colmenares, J. D. O'Connell III and R. M. Stroud, PLoS Biol., 2003, 1, e72.

29 M. O. Jensen, S. Park, E. Tajkhorshid and K. Schulten, Proc. Natl. Acad. Sci. U. S. A., 2002, 99, 6731-6736.
30 J. S. Hub and B. L. de Groot, Proc. Natl. Acad. Sci. U. S. A., 2008, 105, 1198-1203.

31 J. Henin, E. Tajkhorshid, K. Schulten and C. Chipot, Biophys. J., 2008, 94, 832-839.

32 D. Lu, P. Grayson and K. Schulten, Biophys. J., 2003, 85, 2977-2987.

33 I. Kosztin and K. Schulten, Phys. Rev. Lett., 2004, 93, 238102.

34 T. J. Dolinsky, J. E. Nielsen, J. A. McCammon and N. A. Baker, Nucleic Acids Res., 2004, 32, W665-W667.

35 M. H. Olsson, C. R. Sondergaard, M. Rostkowski and J. H. Jensen, J. Chem. Theory Comput., 2011, 7, 525-537.

36 C. Kandt, W. L. Ash and D. P. Tieleman, Methods, 2007, 41, 475-488.

37 M. J. Abraham, T. Murtola, R. Schulz, S. Páll, J. C. Smith, B. Hess and E. Lindahl, SoftwareX, 2015, 1-2, 19-25.

38 R. B. Best, X. Zhu, J. Shim, P. E. Lopes, J. Mittal, M. Feig and A. D. Mackerell Jr, J. Chem. Theory Comput., 2012, 8, 32573273.

39 H. J. Berendsen, J. v. Postma, W. F. van Gunsteren, A. DiNola and J. Haak, J. Chem. Phys., 1984, 81, 3684-3690.

40 G. Bussi, D. Donadio and M. Parrinello, J. Chem. Phys., 2007, 126, 014101.

41 B. Hess, H. Bekker, H. J. C. Berendsen and J. G. E. M. Fraaije, J. Comput. Chem., 1997, 18, 1463-1472.

42 T. Darden, D. York and L. Pedersen, J. Chem. Phys., 1993, 98, 10089.

43 N. Michaud-Agrawal, E. J. Denning, T. B. Woolf and O. Beckstein, J. Comput. Chem., 2011, 32, 2319-2327.

44 O. S. Smart, J. G. Neduvelil, X. Wang, B. Wallace and M. S. Sansom, J. Mol. Graphics, 1996, 14, 354-360.

45 W. Humphrey, A. Dalke and K. Schulten, J. Mol. Graphics, 1996, 14, 33-38.

46 B. Efron and R. Tibshirani, Stat. Sci., 1986, 1, 54-75.

47 M. K. Scherer, B. Trendelkampschroer, F. Paul, G. Pérezhernández, M. Hoffmann, N. Plattner, C. Wehmeyer, J. H. Prinz and F. Noé, J. Chem. Theory Comput., 2015, 330, 341-445.

48 B. Trendelkamp-Schroer, H. Wu, F. Paul and F. Noe, J. Chem. Phys., 2015, 143, 174101.

49 F. Noé, Preprint, 2015.

50 S. Röblitz, Advances in Data Analysis and Classification, 2013, 7, 147-179.

51 E. Weinan and E. Vanden-Eijnden, J. Stat. Phys., 2006, 123, 503-523.

52 P. Metzner, C. Schutte and E. Vanden-Eijnden, Multiscale Model. Simul., 2009, 7, 1192-1219.

53 F. Noe, C. Schutte, E. Vanden-Eijnden, L. Reich and T. R. Weikl, Proc. Natl. Acad. Sci. U. S. A., 2009, 106, 19011-19016.

54 J. H. Prinz, H. Wu, M. Sarich, B. Keller, M. Senne, M. Held, J. D. Chodera, C. Schutte and F. Noe, J. Chem. Phys., 2011, 134, 174105.

55 D. Lu, P. Grayson and K. Schulten, Biophys. J., 2003, 85, 2977-2987.

56 M. Senne, B. Trendelkamp-Schroer, A. S. J. S. Mey, C. Schutte and F. Noe, J. Chem. Theory Comput., 2012, 8, 2223-2238.

57 K. B. Heller, E. C. Lin and T. H. Wilson, J. Bacteriol., 1980, 144, 274-278. 